\title{
A novel technology for investigating the dynamics of infant perseverative reaching
}

\author{
JiNG Feng \\ Indiana University, Bloomington, Indiana \\ JOHN SPENCER \\ University of Iowa, Iowa City, Iowa \\ GrEgor SCHÖNER \\ Institut für Neuroinformatik, Bochum, Germany \\ Melissa W. Clearfield \\ Whitman College, Walla Walla, Washington \\ AND \\ ESTHER THELEN \\ Indiana University, Bloomington, Indiana
}

\begin{abstract}
We designed and tested a novel technology that enables us to capture the entire stream of behavior in perseverative reaching tasks. Beyond fitting the criteria of the conventional A-not-B task, the novel technology gives us better access to the core features of perseverative reaching, such as timing, behavior history, and reinforcement. The technology allows us to quantitatively manipulate reinforcement characteristics, to accurately program onsets, delays, and stimulus durations as well as locations and salience of the targets, and to automatically record the number of reaches to each target and compute timing. We did a validation study on this new technology, investigating the influence of a number of novel factors on perseverative reaching. We replicated the A-not-B phenomenon and associated developmental changes. In addition, results demonstrated that the new technology offers insights into infants' behavior beyond the first reaching action. Moreover, data collection is fully automated, yielding precise, quantitative data. Future studies using this technology will capitalize on the salient advantages of this technology to more precisely measure goal-directed actions and understand the development of reaching skill.
\end{abstract}

Perseverative reaching in infants, typically assessed in the Piagetian A-not-B task, has puzzled developmental psychologists since Piaget's (1954) first report of the phenomenon. Since that time, it has been examined in numerous studies with many variations (e.g., Marcovitch \& Zelazo, 1999; Munakata, 1998; Wellman, Cross, $\&$ Bartsch, 1986). The canonical form of the task is as follows: An infant sits in front of two identical hiding locations (A and B) covered by cloths or lids. The infant watches as an experimenter hides a small object under one of the locations. After a brief delay, the infant is allowed to reach until she obtains the object. In some cases this occurs in a relatively straightforward way: After a few seconds, infants reach out, lift the relevant lid or cloth, and retrieve the object. Other times, infants show more variable behaviors. For instance, they might lift the lid, play with it, put it down, grab the other lid, put this down, and, finally, grab the object from the original hiding location.
The hiding and search events at Location A are repeated a number of times, and then the infant watches as the experimenter hides the object at Location B. If a delay of a few seconds is imposed between hiding and search, 8- to 12-month-old infants reliably reach back to Location A even though they saw the object hidden at Location B.

According to Piaget (1954), the A-not-B error was indicative of the limited nature of infants' object concept; specifically, infants associate the object with a particular location and associate the location with the action made to retrieve the object from that location. Although many researchers since Piaget have put forth explanations regarding infants' knowledge of objects (Baillargeon \& DeVos, 1991; Bertenthal, 1996; Diamond, 1990; Munakata, McClelland, Johnson, \& Siegler, 1997) or spatial memory abilities (Acredolo, 1985; Bremner, 1978; Bremner \& Bryant, 1977; Wellman et al., 1986), current research has shown that hidden objects are not necessary to elicit perse-

J. Feng, jfeng@indiana.edu 
verative reaching. For example, Butterworth (1977) showed that infants perseverated even when the "hidden" object was covered with a transparent lid and thus, still visible. Moreover, Smith, Thelen, Titzer, and McLin (1999) demonstrated perseverative reaching in 8- and 10-month-old infants reaching to one of two identical but visible targets. The experimenter simply directed the infant's attention to one of the two lids by picking it up and waving it. Infants reached consistently for the A lid when it was waved, and continued to reach for the A lid even when the B lid was waved. Therefore, Smith et al. argued, it is the process of reaching to a visual target, not the process of remembering hidden objects, that creates perseverative reaching.

To understand this process of goal-direct reaching, Thelen and colleagues (Smith et al., 1999; Thelen, Schöner, Scheier, \& Smith, 2001) proposed that the A-not-B error and its puzzling contextual variations can be explained by the coupled dynamics of the ordinary processes of goaldirected actions: looking, planning, reaching, and remembering. They developed a dynamic field theory and model that both simulates the known A-not-B effects and offers novel predictions that match experimental results (see Clearfield, Diedrich, Smith, \& Thelen, 2006; Diedrich, Thelen, Smith, \& Corbetta, 2000). In particular, these researchers showed that various factors (stimulation toward one side, history of previous actions, perceptual layout of scene) may influence the side to which infants reach. These insights have led to new questions about perseverative reaching in light of data showing that a variety of task factors influence perseverative reaching.

\section{Timing}

This is well known with respect to the delay between hiding and search (Diamond, 1985; Harris, 1973), but the dynamic field theory predicts that the trial and intertrial duration also matter. The trial duration determines how long infants are exposed to the stimulus, which affects the activation level (and therefore memory strength) for that location. Moreover, the intertrial interval determines the decay of memory traces, with longer intertrial intervals leading to more decay and thus weaker memories for a particular location. In previous studies, trial duration and intertrial intervals were not controlled. Controlling these parameters would lead to insights about the precise timing dynamics of perseveration.

\section{Number of Reach Repetitions to the A Location}

Recent experimental findings (Diedrich, Thelen, Smith, \& Corbetta, 2000; Marcovitch \& Zelazo, 1999; Marcovitch et al., 2002; Smith et al., 1999) showed that there is a strong tendency to reach to A after the infant has already reached to A once and then a persisting memory of Location $\mathrm{A}$ after repeated reaches to $\mathrm{A}$. This persistent memory actually determines the likelihood of the infant's reaching perseveratively (i.e., making the A-not-B error). However, reaches to A might happen more than once on a trial. Previous studies only reported the location of the first reach on each trial and ignored the subsequent reaches. It may be necessary to consider the contribution of all reaches to perseveration.

\section{Spontaneous Errors (Infants' Spontaneous Reaches to B When Cued to A)}

Spontaneous reaches to B during the A trials reduce the accumulated memory strength for A, thus generating less attraction to $\mathrm{A}$ and more tendency to $\mathrm{B}$. Indeed, infants who had reached spontaneously to B when cued to A were more likely to reach to B on the B trials (Diedrich et al., 2000). Given this result, we suspect that the entire reaching history might matter; in other words, every single reach that the infant performs should be analyzed. Yet, most research on the A-not-B error only accounts for the first action to each location on each trial. Automated recording of reaching history would provide us with more comprehensive information on infants' entire motor history and thus may provide more insight into infants' perseverative reaching.

\section{Reinforcement}

The reward contingencies in the task might also play a role in eliciting perseveration, since reinforcement is a part of the continuous stream of reaching behavior. Most researchers assumed that obtaining the toy or the lid was a reward which increased the likelihood of the infants repeating that action. However, the influence of reinforcement on perseveration is not well understood because it has never been systematically manipulated.

The factors listed above all paint a similar picture - the presence or absence of perseverative reaching hinges critically on a continuous stream of action in context. To fully understand what contributes to perseverative reaching in infancy, it is necessary to investigate the entire stream of action. Nonetheless, there is currently no tool to capture this stream of actions. Thus, in the present study, we describe a novel technology, the I-Box or Infant-Box, that allows quantification of the stream of action in infant reaching tasks and examines the dynamics of perseverative reaching using this technology. In particular, we designed and constructed two identical target boxes so that the targets were always visible, the reinforcement could be precisely manipulated, and the entire behavioral history could be automatically recorded. Although we did not study the A-not-B error per se, we did study perseveration in goal-directed reaching using a paradigm identical to the traditional A-not-B paradigm. Our results show that the novel technology offers better access to core features of perseverative reaching, such as timing, behavior history, and reinforcement.

\section{THE I-BOX TECHNOLOGY}

In an effort to design a general technology that would both quantify the stream of action in perseverative reaching tasks and allow for ties to the many studies of the A-not-B phenomenon, we began by abstracting the fundamental aspects of A-not-B tasks from dozens of previously published reports. Despite minor task variations, nearly all A-not-B tasks share the following characteristics. (1) Task input: There are two visually identical reaching targets. (2) Specific input to A: Infants are cued to reach to Target A. (3) Repeated reaches to A: Infants reached to A several times (generally between four and six times). (4) Specific input to B: Infants are cued to reach to Target B. (5) Delay: 
There is a short delay. (6) A-not-B error: When infants are permitted to reach after the delay, they reach back to the original Target A, even though they are cued to Target B.

Our I-Box technology affords access to all of these fundamental elements of the A-not-B task. In addition, our paradigm allowed us to manipulate timing and reinforcement in a more precise and quantitative way. We next present technical details of our new technology for investigating perseveration. Then, we present the results of a longitudinal study of perseverative reaching in which we validate the new technology. Finally, we discuss the advantages and limitations of our novel technology as well as its potential applications.

\section{Details of the I-Box}

We designed and constructed two identical target boxes, both black in color, $21 \times 18.5 \times 8 \mathrm{~cm}$. Each box has a large orange illuminable button $(6 \mathrm{~cm}$ diameter, $1 \mathrm{~cm}$ above the surface of the box) in the center and six colored lights $(1.5 \mathrm{~cm}$ in diameter and $7 \mathrm{~cm}$ from the center of box) that surround the central button (Figure 1). When infants press the button, the colored lights flash around the button and the box emits enticing sounds. The boxes are constructed so that the following features of stimulus and reward can be computer-controlled: the brightness and onset and offset of the illuminated button, the onset and offset of colored lights, the onset and offset of sound, and the force necessary to trigger the reward. Moreover, the number, timing, and force profile of the buttonpresses are fed into a LabVIEW data acquisition program and recorded on a computer. This allows us to present stimuli with varying schedules, delays, and salience levels, while simultaneously recording rich data about infant responses. Stimulus presentations are contingent on infant responses. This design offers flexibility in setting up various experiments on perseveration. Infants can be trained to reach to one target repeatedly or to alternate between targets. The experimental task can be to reach with hands or to kick with feet, or even just to look at the targets with an experimenter triggering the audio/video reward by keypress when the infant fixates the correct target.

A National Instruments PCI-6025E DAQ board provides the interface (see the input and output in Table 1) between the boxes and the computer. The force applied on the central button is measured using a strain gauge and sent to the computer as an analog input signal in the range of 0 to $10 \mathrm{~V}$, which corresponds to 0 to $20 \mathrm{lbs}(1 \mathrm{~V}$ corresponds to $8.9 \mathrm{~N})$. An analog output signal $(0$ to $10 \mathrm{~V})$ from the computer controls the brightness of the central light when it is on. The digital output from the computer contains 8 bits. Bits 1 to 6 control whether each of the six surrounding lights is on or off, respectively. Bit 7 turns the central light on or off. Bit 8 controls the on-off of the speaker installed inside the boxes. A sound file is repeatedly played on the computer using Winamp and sent to the speakers inside the boxes. By turning the speaker on or off, the sound is turned on or off.

A LabVIEW data acquisition program (virtual instrument, or VI) was written to control the boxes and collect data on infants' responses on the button. The main VI is an event handler that detects the users' selection of the following actions: input participant information, setup

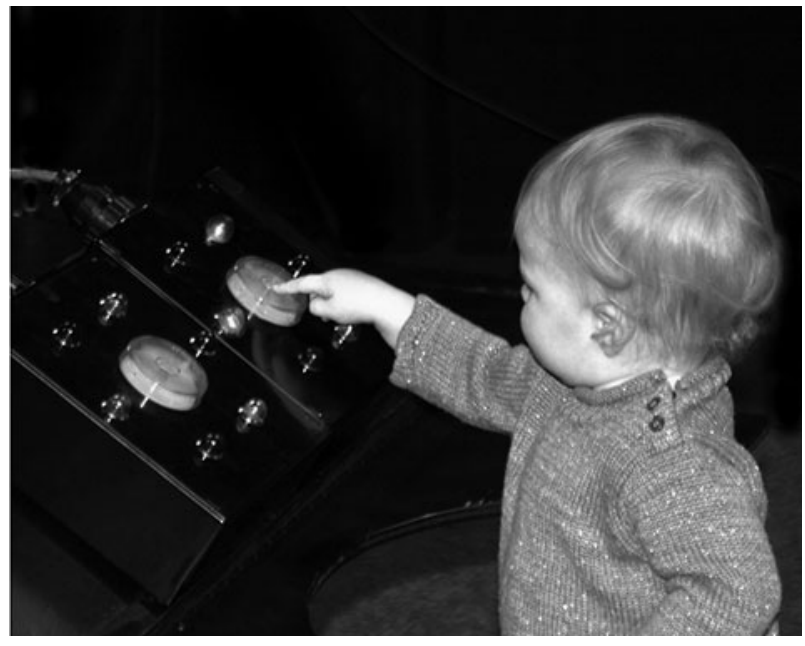

Figure 1. The boxes and the experiment setup. Two customdesigned boxes, each with a large central button and surrounding colored lights. The correct target is cued by a flashing light under the large central button. When the cued button is pressed, surrounding lights flash and an interesting sound emits from the box.

experiment, run experiment, or exit the program. As soon as a selection is made, the corresponding subVI is called. In the Input Participant Information subVI, participant's information such as name, ethnicity, date of birth, date of the experiment, age of the participant at testing, and so on can be saved in a text file. The following can be set up in the Setup Experiment SubVI: sampling rate, central light brightness for each box, central light illuminating pattern (flashing/steady), central light flashing rate, surrounding light on-off, surrounding lights flashing rate, number of A trials, number of B trials, the sequence of A and B trials, trial duration, and time delay before each $\mathrm{B}$ trial. To cue infants to one of the targets, the on-off schedule and the brightness of the central light can be set up to create presentation stimuli with varying schedule, delay, and salience levels. To reward buttonpresses, the surrounding lights can be set up to be illuminated in varying schedules, and the speaker inside a specific box can be turned on to play music. Since the music being played is a sound file on the computer, different music can be selected.

In the Run Experiment subVI, after a Boolean button labeled as "Demonstrate" is clicked, the demonstration phase (where the experimenter demonstrates the buttonpressing and the reward of flashing lights and music) of the experiment starts (see Figure 2). Another click of the "Demonstrate" button terminates the demonstration phase and starts the test phase of the trial (for a detailed example, see procedure below). While the duration of demonstration is indefinite, the duration of the test phase (trial duration) is fixed. There is a force chart panel displaying the online force applied on the buttons. Two LED lights above the force chart panel indicate the on-off states of the buttons. The current trial number, the elapsed time on the current trial, and the number of backlogged samples (which happens when the speed of data recording is slower than the speed of data acquisition) are displayed and updated online. 
Table 1

Input and Output Assignments for the

National Instruments PCI-6025E DAQ Board

\begin{tabular}{|c|c|}
\hline \multicolumn{2}{|c|}{ Analog Input } \\
\hline AD0 & Box A response button strain gauge, $0-10 \mathrm{~V}, 0.5 \mathrm{~V}$ per pound \\
\hline AD1 & Box B reponse button strain gauge, $0-10 \mathrm{~V}, 0.5 \mathrm{~V}$ per pound \\
\hline \multicolumn{2}{|c|}{ Analog Output } \\
\hline DA0 & Box A response button light brightness, $0-10 \mathrm{VDC}$ \\
\hline DA1 & Box B response button light brightness, $0-10 \mathrm{VDC}$ \\
\hline \multicolumn{2}{|c|}{ Digital Output } \\
\hline PA0 & Box A light 1,0 for On, 1 for Off \\
\hline PA1 & Box A light 2, 0 for On, 1 for Off \\
\hline PA2 & Box A light 3, 0 for On, 1 for Off \\
\hline PA3 & Box A light 4, 0 for On, 1 for Off \\
\hline PA4 & Box A light 5,0 for On, 1 for Off \\
\hline PA5 & Box A light 6,0 for On, 1 for Off \\
\hline PA6 & Box A response button light, 0 for On, 1 for Off \\
\hline PA7 & Box A speaker, 0 for On, 1 for Off \\
\hline PB0 & Box B light 1,0 for On, 1 for Off \\
\hline PB1 & Box B light 2, 0 for On, 1 for Off \\
\hline PB2 & Box B light 3, 0 for On, 1 for Off \\
\hline PB3 & Box B light 4, 0 for On, 1 for Off \\
\hline PB4 & Box B light 5,0 for On, 1 for Off \\
\hline PB5 & Box B light 6,0 for On, 1 for Off \\
\hline PB6 & Box B response button light, 0 for On, 1 for Off \\
\hline PB7 & Box B speaker, 0 for On, 1 for Off \\
\hline
\end{tabular}

Whether the button is pressed is calculated online in the LabVIEW program based on the force applied to the button. We set up a threshold appropriate for most infants at the experimental age, so that only actual buttonpresses would be rewarded. If the threshold were too low, even light brushes of the button would trigger the response. If the threshold were too high, infants would need to press the button hard to get the response. Neither case is ideal because we want to give the reward for infants' clear button-pressing only, as opposed to contact in the course of flapping arm movements. After piloting on a number of infants, we adopted the following algorithm: (1) The force signal is smoothed using a 50-msec moving average window; (2) the moving averaged signal of force is squared to detect changes in signal more efficiently; and (3) the squared signal is compared with a high threshold of 4 square volts and a low threshold of 1 square volt. We sampled the continuous force data at $100 \mathrm{~Hz}$. The button is considered on (pressed) if (a) the current sample is higher than the high threshold, (b) the squared signal has been higher than the low threshold for more than $50 \mathrm{msec}$, or (c) the button was on in the previous sample and the squared signal has been lower than the low threshold for less than $50 \mathrm{msec}$. Otherwise, the button is considered off (released).

\section{VALIDATION STUDY}

\section{Method}

Participants. Twenty-six healthy infants, 14 boys and 12 girls, participated in this study. They were recruited from Bloomington and Indianapolis, Indiana. Each infant was tested twice, at 35 weeks and 45 weeks, respectively. Four boys and 2 girls missed one of the sessions due to sickness, vacation, or relocation. Their data were not included in the data analysis. Parents of the infants gave informed consent and received a small gift for participating.

Procedure. The two target boxes were placed side by side (the distance between the centers of the buttons was $18.5 \mathrm{~cm}$ ) on a tilted board ( $30^{\circ}$ relative to horizontal) that was fixed on a large, black, padded table (Figure 1). There was a semicircular notch $(40 \mathrm{~cm}$ diameter) cut out of the front of the table, in which the infant could sit on her parent's lap facing forward and across the table from the experimenter. The parent sat on a rolling chair, which allowed the parent to move the infant away from the boxes easily.

After the parent completed the consent documents and the infant was comfortable in the lab, the parent and infant sat down in front of the boxes, and the infant was given a short period of time to get familiar with the boxes. The infant could touch and play with the boxes freely. All the lights on the box were off, and the boxes did not give any response during this familiarization period.

Next, we began the experimental trials. Each trial consisted of two phases: demonstration and test. In the demonstration phase, the experimenter demonstrated the buttonpress by pressing the button on the cued side three or more times until the infant focused his or her attention in the appropriate direction. During this time, the parent's chair was far enough away from the boxes that the infant could not reach them. This was followed by a 15 -sec test phase. When the testing phase started, the parent was instructed to immediately roll the chair forward to the edge of the table so that the boxes were within the infant's comfortable reaching distance. The infant's engagement with the boxes was recorded only during the testing period of each trial.

The experimenter cued the $\mathrm{A}$ target six times (Trials A1-A6) and then the B target twice (Trials B1-B2), as in the canonical A-not-B task (e.g., Smith et al., 1999). The side (left or right) cued as A was randomly assigned to each infant and counterbalanced across participants. The A and B trials were identical, with two exceptions: (1) A different location was cued, and (2) the testing phase started immediately after the demonstration on A trials, but after a 3-sec delay on B trials (as is the case in many other A-not-B studies; see, e.g., Wellman et al., 1986). Note that although the delay period differed on A and $\mathrm{B}$ trials, the test phase was identical, with $15 \mathrm{sec}$ on both trial types. On Trials A1 and A2, the parent was instructed to hold the infant's arm and help the infant to press the cued button. On later trials, the parent was asked not to talk, point, or interact in any way that would influence or distract the infant; however, the parent was allowed to say encouraging words when the infant pressed the button.

Only the correct target box gave the infant feedback during both the demonstration and the testing phases of each trial. During the 3 -sec 

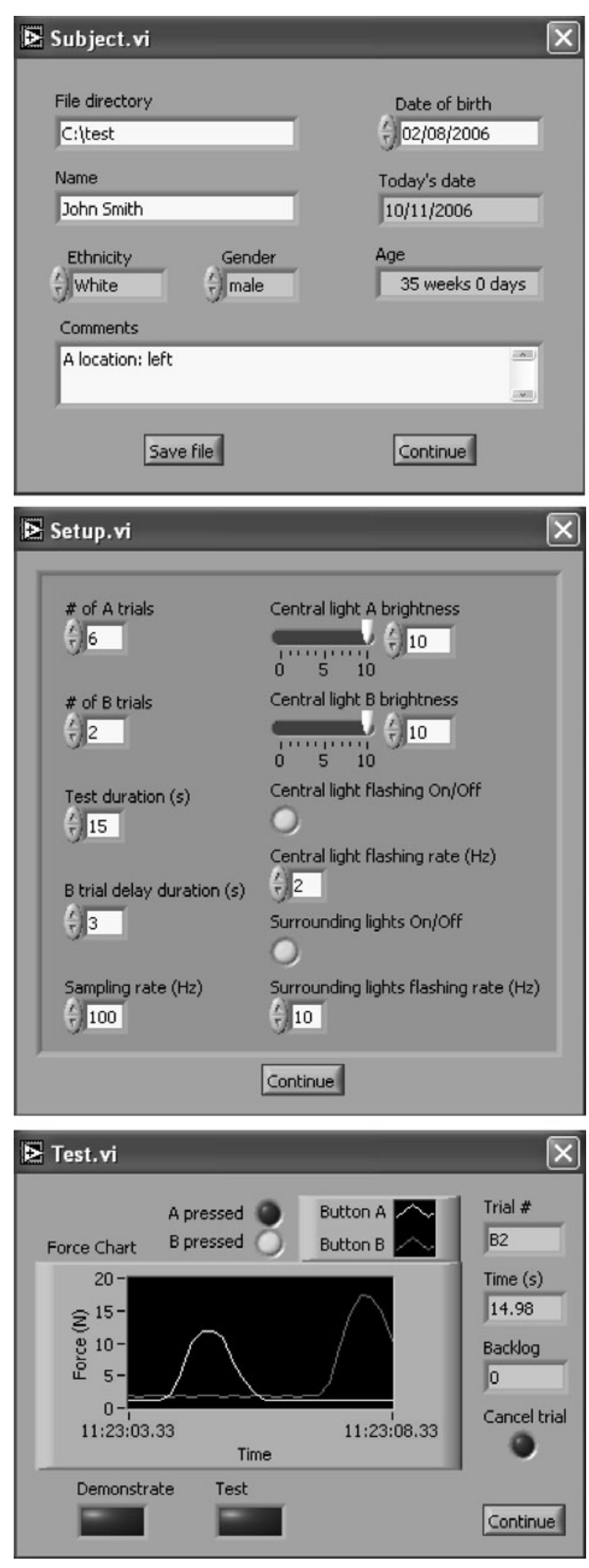

Figure 2. Sample screen shots of the LabVIEW program for running the experiment. delay on each B trial, all lights on both boxes were off, and the boxes did not give any response. The feedback from the correct box was as follows: When the button was not pressed, the central light flashed at the rate of $2 \mathrm{~Hz}$ to cue the infant to the correct target. The brightness of the central lights was set at the brightest level. When the button was pressed, the central light became steadily illuminated, and the surrounding lights flashed in a roll at the rate of $10 \mathrm{~Hz}$. At the same time, an interesting sound was played from the speaker inside the correct target box. A sound effect wave file, which had proved to be attractive to most infants, was used for this experiment. Only the correct target box gave feedback to buttonpresses. The other box did not give any response, even when pressed. However, information (timing and force profile) about buttonpresses on both boxes during the testing period was sampled at $100 \mathrm{~Hz}$ and recorded into a data file.

The entire experimental session was videotaped with a video camera mounted in front of the infant.

Data analysis. Since Trials A1 and A2 were helped by parents, we only analyzed Trials A3-A6 and B1-B2.

To validate the I-Box technology, we compared data from the boxes to standard behavioral coding. In particular, we coded from videotapes whether the infant touched the button before the first buttonpress recorded by the computer. We also coded which box was touched and whether the location of the first touch (A or B) was consistent with that recorded on the computer. On approximately $50 \%$ of all trials that were randomly selected, two observers coded the first touch of the button for reliability purposes. Percentage of agreement was $86.7 \%$. Observers' records of the latency from the start of the trial to the first touch of button had a correlation of 0.95 .

Based on the data recorded on the computer, we calculated the following variables: (1) percentage of infants who pressed the correct button in the first buttonpress on each trial; (2) number of buttonpresses $\times$ trial, averaged across infants; (3) percentage of correct buttonpresses $\times$ trial, averaged across infants; (4) average amount of switching between A and B presses, measured as a percentage of the total number of presses $\times$ trial; and (5) duration and force of buttonpresses. Note that in the vast majority of previous studies of perseverative reaching, only Variable 1 is examined.

In determining the number of buttonpresses, the press on each button was counted independent of its timing relationship with the presses on the other button. In other words, when both buttons were pressed at about the same time, they were registered as two separate buttonpresses. The exact time difference in the onset of the seemingly simultaneous buttonpresses was detected by the computer, as the data was sampled and recorded at $100 \mathrm{~Hz}$. To estimate how frequently bilateral buttonpresses happened, we defined a bilateral buttonpress to be a buttonpress that happened within 0.5 seconds of a press on the other button. Then we calculated the percentage of bilateral buttonpresses of all trials for each session of each infant. Bilateral buttonpresses did not happen very often, and the percentage was not significantly different between 35 weeks and 45 weeks $[4.3 \% \pm 0.9 \%$ vs. $5.9 \% \pm 1.2 \% ; t(20)=-1.624$, n.s. $]$.

\section{Results}

In this section, we first confirmed the match between the computer-recorded data and videotaped responses for the first reach of each trial. Then, we analyzed infants' first reach on each trial and compare these data to previous studies of infant perseverative reaching. Next, we analyzed the impact of infants' reaching history on the entire trial. Finally, we explored some of the more refined measures that our technology can capture, such as duration and force of the buttonpress.

To confirm that the computer captured goal-directed reaching to the buttons, we coded infants' first touches of the buttons from the videotape and compared the re- 
sponse with the buttonpresses recorded on the computer. Overall, the first press recorded by the computer matched the videotape on $92.1 \%$ of the trials. Thus, there was very good agreement overall. The $7.9 \%$ of all trials where there was a disagreement fell into two categories: (1) On 5.8\% of all trials, infants touched the button before the first computer-recorded buttonpress, and the location (A or B) of the first button touch was not consistent with that of the first recorded buttonpress. (2) On $2.1 \%$ of all trials, infants touched one of the buttons, but there was no buttonpress recorded on the computer.

Closer examination of the timing of the responses where there was a match between the video and the computerrecorded responses showed a timing discrepancy on $10.9 \%$ of all trials: On these trials, infants touched the button before the first recorded computer buttonpress, but later touched the same button more forcefully, making the location of the first button touch consistent with the first recorded buttonpress. Recall that we intentionally set the force threshold to capture only clear buttonpressing, not random touching. It is possible that this threshold was a bit too high. That said, it is important to emphasize two aspects of these reliability results. First, the overall agreement was quite good (92.1\%). Second, the I-Box opens up new avenues for investigating the characteristics of infants' movements - including details of force production - that are not available using video coding. Given the overall level of agreement between the computer-recorded and video-coded responses, we conclude that the I-Box data were highly reliable. Therefore, in the rest of this article, we only report results from data recorded on the computer.

In a typical A-not-B experiment, a trial is over once the infant gets the lid or toy, and only the initial reach to the lid or toy on each trial is analyzed. To compare our data with typical A-not-B data, we analyzed the first buttonpress on each trial and calculated the percentage of infants who pressed the correct button (Button A on A trials and Button $\mathrm{B}$ on $\mathrm{B}$ trials) on each trial. A binomial test showed that the percentage of infants who pressed the correct button on A trials was significantly above chance $(p<.05)$ at both 35 weeks and 45 weeks (Figure 3). However, the percentage of infants who pressed the correct button on B trials was only at chance. Thus, infants perseverated in this task. Note too the trend toward greater perseveration at 35 weeks, which is consistent with previous studies (e.g., Diamond, 1985).

In both our paradigm and in classic A-not-B studies, it is possible for infants to reach more than once on each trial. However, no previous studies have measured how often infants actually reach to each location, despite evidence that where and how often an infant has reached in the past determines the likelihood of a perseverative reach. On average, infants pressed the buttons $6.8 \pm 5.0$ times per trial at 35 weeks and $8.7 \pm 5.5$ times per trial at 45 weeks. A repeated measures ANOVA revealed that this age effect was significant $[F(1,20)=5.97, p<.05]$. There was no effect of trial, nor an interaction. Therefore, any differences in performance between the $\mathrm{A}$ and $\mathrm{B}$ trials were not due to differences in the overall number of buttonpresses.

Although infants pressed the correct button most of the time on A trials ( $66 \%$ to $91 \%$ ), they pressed the correct button

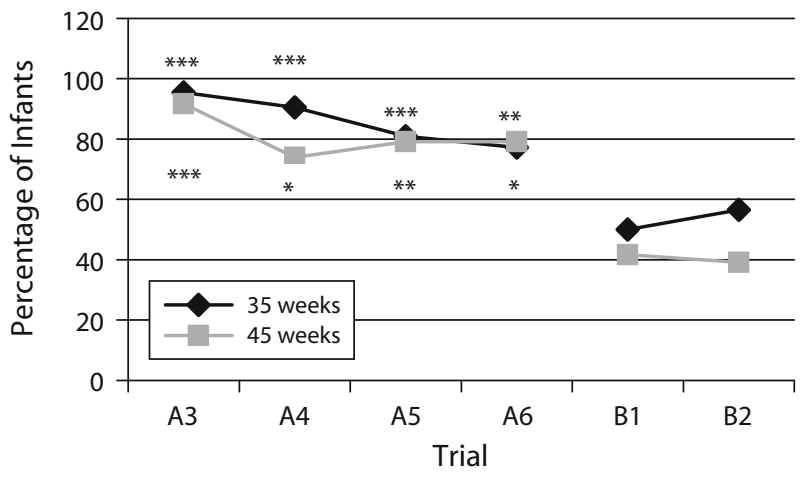

Figure 3. Percentage of infants who pressed the correct button on the first buttonpress on each trial. Significance of binomial test with $50 \%$ test proportion is indicated by stars: $\quad{ }^{*} p<.05 . \quad{ }^{* *} p<$ $.01 .{ }^{* * * *} p<.001$.

at a much lower rate on B trials ( $44 \%$ to $67 \%$; see Figure 4 ). An ANOVA with repeated measures on A trials revealed an effect of age $[F(1,16)=6.378, p<.05]$, with infants pressing the incorrect (B) button more frequently on the A trials at 45 weeks than at 35 weeks. There was no effect of trial, nor an interaction. In addition, we were interested in comparing performance on the last A trial with the first B trial. An ANOVA with repeated measure on Trials A6 and B1 $\times$ age revealed an effect of trial $[F(1,18)=14.883, p<.01]$, with the infants pressing the correct button at a significantly higher rate on Trial A6 than on Trial B1. There was no effect of age nor an interaction. Therefore, when we analyzed all reaches during a trial, infants showed robust perseveration.

An ANOVA with repeated measures on Trials B1 and B2 $\times$ age showed a trend for an age effect $[F(1,19)=$ $3.744, p=.068]$. We investigated the age effect further by looking at the percentage of correct buttonpresses on all B trials. A Student's $t$ test showed a significant age effect, with the infants pressing the correct button on $\mathrm{B}$ trials at a higher rate at 45 weeks $(66.4 \% \pm 22.2 \%)$ than at 35 weeks $(44.1 \% \pm 29.2 \%, p<.01)$. This is again consistent with previous studies on infant perseverative reaching in the A-not-B task (e.g., Diamond, 1985).

One factor that contributes to perseveration is motor history, including both the number of reach repetitions to

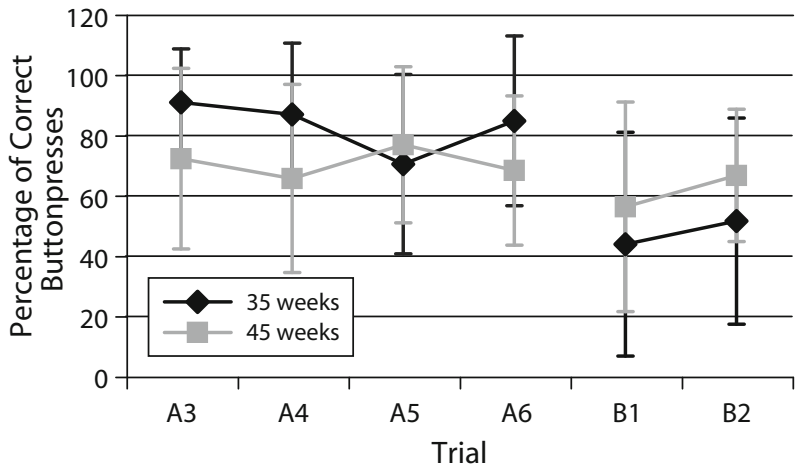

Figure 4. Percentage of correct buttonpresses by trial, averaged across infants. 
the $\mathrm{A}$ location and the number of reaches to the $\mathrm{B}$ location when cued at Location A (i.e., spontaneous errors). We captured this potential back and forth between the A and B locations by computing the percentage of switching for a trial. Figure 5 illustrates an infant's switching between the two locations on an A trial. In this example, the infant switched from Location A to B at the 4th and the 8th buttonpresses and switched from B back to $\mathrm{A}$ at the 5 th and 9 th buttonpresses. Among the 10 buttonpresses, the infant switched four times between locations. Thus, the percentage of switching for this trial was $40 \%$. Figure 6 shows the percentage of switching between locations $\mathrm{A}$ and $\mathrm{B} \times$ trial, averaged across infants. Infants made more switches at 45 weeks $(22.0 \% \pm 23.1 \%)$ than at 35 weeks $(12.4 \% \pm$ $17.8 \%)$. A repeated measures ANOVA revealed that this age effect was significant $[F(1,21)=5.791, p<.05]$. There was also an effect of trials $[F(5,105)=3.224, p<.05]$, but no interaction. Post hoc $t$ tests (Bonferroni-corrected) revealed that infants made significantly more switches on Trial B2 $(25.5 \% \pm 21.6 \%)$ than on Trial A3, the first trial after training $[12.8 \% \pm 20.4 \%[t(43)=12.68, p<.003]$. This shows that early in the session (Trial A3), infants followed the cue and made few switches; but later in the session (B2), presses were more variable, resulting in many switches. We suspect that the high percentage of switching later in the study reflected increasing exploration of the boxes.

Unlike conventional A-not-B tasks, our paradigm enabled us to record all of the infants' reaches to each target throughout each 15 -second trial. Not only does this allow us to record a detailed motor history for each infant, but we can also apply more sensitive statistical measures to that motor history (such as ANOVA and multiple regression), compared to the standard binomial test typically used in A-not-B studies with one response recorded per trial. We analyzed how each infant's own repetition of reaches to a location predicted his/her own subsequent behavior by running a multiple regression. The dependent variable was the number of presses to A on B trials (an index of the degree of perseveration), and the three independent variables were the total number of buttonpresses on all trials, the percentage of presses to A on A trials, and age. The multiple regression (Table 2) shows that the number of buttonpresses on all trials strongly predicted the number of A presses on

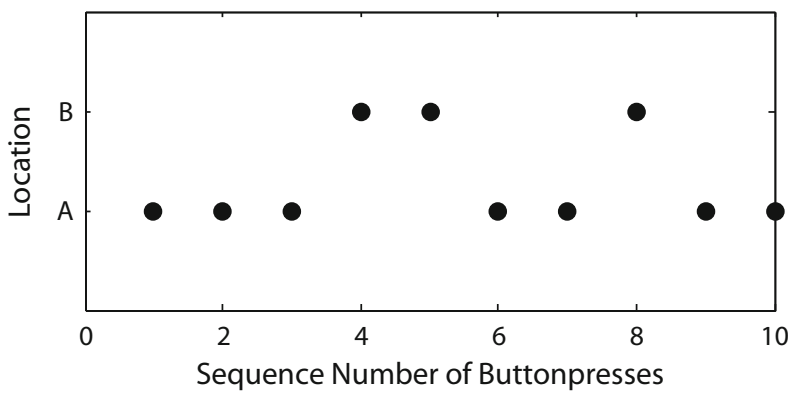

Figure 5. An illustration of switches between locations on a single A trial for 1 infant. Note the spontaneous errors made at reaches 4,5 , and 8 . In this case, the infant switched 4 times between $A$ and $B$ locations out of 10 buttonpresses total, resulting in switches $40 \%$ of the time.

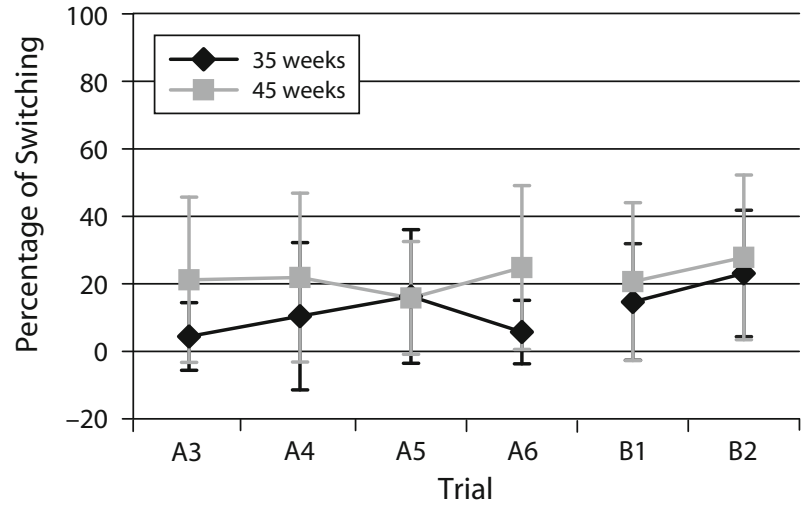

Figure 6.Average amount of switching between $A$ and $B$ presses, measured as a percentage of the total number of presses by trial.

B trials. The number of buttonpresses on all trials generally reflects how active an infant was pressing the buttons. The more active infants pressed button A more on both $\mathrm{A}$ and $\mathrm{B}$ trials. However, even after this contribution is controlled, a higher percentage of A presses on A trials significantly predicted more A presses on B trials (A-not-B error). This adds novel, rigorous statistical evidence that motor history is critical to perseveration.

With the I-Box, it is possible to explore more refined measures of infant behavior, such as duration of buttonpress and force applied on the button. An ANOVA with repeated measures on the mean duration per buttonpress revealed an effect of age $[F(1,19)=8.74, p<.01]$. The mean duration per press was significantly longer at 45 weeks $(0.83 \pm 0.71 \mathrm{sec})$ than at 35 weeks $(0.59 \pm$ $0.53 \mathrm{sec}$ ). However, there was no effect of trials nor an interaction. An ANOVA with repeated measures on the mean force during a buttonpress also revealed an effect of age $[F(1,20)=4.87, p<.05]$, with 45 -week-old infants pressing the button significantly harder $(0.18 \pm 0.09 \mathrm{~N})$ than did 35 -week-old infants $(0.15 \pm 0.06 \mathrm{~N})$. Again, there was no effect of trials nor an interaction. Therefore, older infants pressed the button longer and with more force. Nevertheless, these differences did not play a major role in the perseverative tendencies we report.

\section{DISCUSSION}

The results of our validation study replicated the central findings of the perseverative reaching literature, including developmental changes in perseverative tendencies. Critically, we also extended these findings by looking at infants' actions on the entire trial and how the history of actions on previous trials affected perseveration. We found that infants perseverated in this task at comparable rates to other studies (e.g., Smith et al., 1999). Moreover, we replicated the finding that reaching on the B trials is determined, at least in part, by the number of reaches to A on the A trials (e.g., Marcovitch \& Zelazo, 1999). Repeated reaches to A built a strong tendency to reach to A on B trials, perseveration. In contrast, spontaneous errors or incorrect reaches to $\mathrm{B}$ on $\mathrm{A}$ trials formed a strong $\mathrm{B}$ attraction, resulting in 
Table 2

Results of a Multiple Regression Analysis

\begin{tabular}{clcc}
\hline & \multicolumn{1}{c}{ Independent Variables } & Standardized & Coeff. \\
Dependent Variable & \multicolumn{1}{c}{ Sig. } \\
\hline Number of A presses on B trials & Total number of buttonpresses on all trials & .100 & .000 \\
& Percentage A presses on A trials & .569 & .048 \\
& Age & .267 & .455 \\
\hline
\end{tabular}

Note-The standardized coefficients reflect unique prediction above and beyond the other predictors and their shared variance.

less perseveration on the $\mathrm{B}$ trials. In addition, the infants perseverated less at 45 weeks than at 35 weeks, which is also consistent with early research (e.g., Diamond, 1985). However, we found that at 45 weeks of age, the infants switched locations and explored the I-Box more quickly (and more frequently) than they did at 35 weeks. This could be because their previous exposure to the boxes at 35 weeks induced boredom with the task at the second visit. However, the duration and force data demonstrated that the 45-week-old infants pressed the buttons longer and harder, suggesting that these infants were not simply bored by the procedure. Rather, their frequent switching may reflect exploratory behavior.

The age differences combined with the continuous motor history provide new insight into why perseveration decreases with age. Our data show that infants perseverated less as they got older, not just because they were better able to follow the cue, but because they had made more spontaneous errors during the $\mathrm{A}$ trials as well. By the conventional measure of A-not-B behavior (first response on the switch trial), our data would support previous studies that found that infants improve with age (e.g., Diamond, 1985). Despite differences in the structure of our task compared to those earlier studies, infants' behavior may be similar across studies. Specifically, in Diamond's (1985) task (and others), infants were allowed to reach until they uncovered the toy, which could have happened on the first reach or after many reaches toward the toy, including potential spontaneous errors. The present results indicate that decreases in perseveration cannot simply be attributed to improvement on the task, but, rather must be explained in the context of the full motor history.

Despite multiple explanations for why infants make perseverative reaching errors, most researchers agree that the A-not-B task is manifestly a reaching task. In light of this, the I-Box technology offers many advantages in that it enables us to study goal-directed reaching in a more focused way. Importantly, the boxes are not based on any assumptions about the child's motives with respect to hidden objects. In addition, in the boxreaching task, infants need not grasp anything to get a reward. This has two advantages: (1) Reaching is easier than grasping, and so this paradigm can be used with a wider range of motor skills. (2) The boxes eliminate the awkward wrenching of the toy out of the child's hand for the next trial. Also, the new technology enabled us to capture every buttonpress throughout the entire trial, while selecting an appropriate threshold for determining a clear press. This allowed us to statistically analyze these motor histories with more sensitive statistical measures, such as ANOVAs and multiple regression. Finally, the experimental paradigm can be precisely and quantitatively controlled, and data collection is fully automated. We can accurately program the trial onsets, stimulus duration, and delays, as well as the size, locations, and salience of the targets. We also have better control over reinforcement, in that the boxes give visual and auditory feedback that can be varied in both amount and nature. In addition, when infants alternate between boxes, we can determine the onset of the movement with much more accuracy, since we can program the computer to record number of presses and force applied on each reaching target and to compute timing information.

In addition to goal-directed reaching, the I-Box technology also offers opportunities for many potential areas of research. For example, it can be used in studying memory for action, memory for location, and selection of location in infants too young for the A-not-B task (younger than 8-12 months). If the A-not-B error arises from the general process of goal-directed reaching, then perseveration may not be limited to a particular age in infancy. Indeed, Clearfield et al. (2006) have tested perseverative reaching in 5-month-old infants, and Spencer, Smith, and Thelen (2001) have shown perseveration in 2- to 3-year-old children. Perseveration, thus, should be observable at different points in development, and our technology can be used across a wide age range. As one example, the boxes would allow us to study stimulus-oriented action with other effectors, such as eyes or feet. For instance, Galloway and Thelen (2004) reported that 2- to 3-month-old infants were able to control their legs before their arms. Given the opportunity to interact with toys, infants as young as eight weeks of age were able to do so with the feet a month before they could reach with their hands. We are currently testing younger infants with a perseverative kicking paradigm using the boxes, making it possible to compare the developmental trajectories of perseveration within the upper and lower limbs.

Besides studying goal-directed kicking, the boxes also enable us to compare perseverative looking and reaching. The visual violation-of-expectancy paradigm (Baillargeon \& Graber, 1988) has been used to investigate differences in infants' performance in search and nonsearch A-not-B tasks. However, the search and nonsearch tasks were not directly comparable, in that the looking tasks consisted of complex events such as a car rolling down a track, while the reaching tasks consisted of the ambiguous two-target reaching task. Infants find our flashing boxes to be inter- 
esting visual stimuli; thus, we can carry out both reaching and nonreaching perseveration studies using the same boxes. Infants as young as 3 to 4 months can learn to get a reinforcement signal by simply looking at the boxes, providing us with a more direct comparison of reaching and nonreaching behaviors.

The reaching and pressing movements in our paradigm are more similar to the pointing tasks used in the adult trajectory literature, allowing us to make more direct comparisons between the reaching dynamics of children and adults. For example, it would be interesting to investigate whether infants' reaching to the boxes follows Fitts's Law (Fitts, 1954), which is a model of human psychomotor behavior. Fitts discovered a formal relationship that models speed-accuracy tradeoffs in rapid, aimed movements. According to Fitts's Law, the time to move and point to a target of width $W$ at a distance $A$ is a logarithmic function of the spatial relative error $(A / W)$. Physically interpreted, big targets at a close distance are acquired faster than small targets at a large distance. By varying the size and distance of the two buttons, we can test for speed-accuracy tradeoffs in infants' reaching movement. In addition, the ability to measure the force of the pressing can also contribute to our understanding of such tradeoffs.

Although the I-Box technology has many novel applications, we also found several limitations in the present study. A part of the visual feedback - the flashing of the surrounding lights - was so attractive to the infants that sometimes infants touched the surrounding lights before they pressed the buttons. This exploratory behavior was missed by the computer. Future experiments with this technology should therefore eliminate the surrounding lights, since the center light is sufficiently interesting. Other aspects of our paradigm that need to be improved include trial onset and trial duration. In the current LabVIEW program, a trial starts as soon as the demonstration finishes. On a few trials, the infants did not reach at all. To make our paradigm more comparable to conventional A-not-B tasks, we can modify the program so that a trial starts when the infant presses a button (analogous to infantcontrolled procedures in studies of infant habituation).

In sum, the I-Box technology offers the opportunity to study infants' goal-directed action in a more precisely controlled way. The ability to precisely measure continuous goal-directed movements will provide us with a deeper understanding of the underlying dynamics of movement and motor skill development, shedding light on classic issues in child development.

\section{AUTHOR NOTE}

This study was supported by NIH Grant HD 22830 awarded to E.T. We gratefully acknowledge Christopher Smethurst's original idea for the prototype of I-Box. Special thanks to Dexter Gormley, Dwight Hector, Mike Bailey, Jeff Sturgeon, and Nathan Engle for designing and building the I-Box. In addition, we are grateful to Dexter Gormley, Andrew McKittrick, Gerrian Greenwade, Melissa Mills, Melissa Haro, and Jennie Preuss for their help with data collection and analysis. We also thank Mary Jane Gormley for her assistance editing the documents. Correspondence concerning this article should be addressed to J. Feng, Department of Psychological and Brain Sciences, Indiana University, 1101 East 10th Street, Bloomington, IN 47405 (e-mail: jfeng@indiana.edu).

\section{REFERENCES}

Acredolo, L. P. (1985). Coordinating perspectives on infant spatial orientation. In R. Cohen (Ed.), The development of spatial cognition (pp. 115-140). Hillsdale, NJ: Erlbaum.

Baillargeon, R., \& DeVos, J. (1991). Object permanence in young infants: Further evidence. Child Development, 62, 1227-1246.

Baillargeon, R., \& Graber, M. (1988). Evidence of a location memory in 8-month-old infants in a non-search A-not-B task. Developmental Psychology, 24, 502-511.

Bertenthal, B. I. (1996). Origins and early development of perception, action, and representation. Annual Review of Psychology, 47, 431-459.

Bremner, J. G. (1978). Spatial errors made by infants: Inadequate spatial cues or evidence of egocentrism? British Journal of Psychology, 69, 77-84.

Bremner, J. G., \& Bryant, P. E. (1977). Place versus response as the basis of spatial errors made by young infants. Journal of Experimental Child Psychology, 23, 162-171.

Butterworth, G. (1977). Object disappearance and error in Piaget's Stage IV task. Journal of Experimental Child Psychology, 23, 391-401.

Clearfield, M. W., Diedrich, F. J., Smith, L. B., \& Thelen, E. (2006). Young infants reach correctly in A-not-B tasks: On the development of stability and perseveration. Infant Behavior \& Development, 29, $435-444$.

Diamond, A. (1985). Development of the ability to use recall to guide action, as indicated by infants' performance on A-not-B. Child Development, 56, 868-883.

DiAMOND, A. (1990). Developmental time course in human infants and infant monkeys, and the neural bases of inhibitory control in reaching. In A. Diamond (Ed.), The development and neural bases of higher cognitive functions (pp. 637-676). New York: National Academy of Sciences.

Diedrich, F. J., Thelen, E., Smith, L. B., \& Corbetta, D. (2000). Motor memory is a factor in infant perseverative errors. Developmental Science, 3, 479-494.

FITTS, P. M. (1954). The information capacity of the human motor system in controlling the amplitude of movement. Journal of Experimental Psychology, 47, 381-391.

Galloway, J. C., \& Thelen, E. (2004). Feet first: Object exploration in young infants. Infant Behavior \& Development, 27, 107-112.

HARRIS, P. L. (1973). Perseverative errors in search by young infants. Child Development, 44, 28-33.

Marcovitch, S., \& Zelazo, P. D. (1999). The A-not-B error: Results from a logistic meta-analysis. Child Development, 70, 1297-1313.

Marcovitch, S., Zelazo, P. D., \& Schmuckler, M. A. (2002). The effect of the number of A trials on performance on the A-not-B task. Infancy, 3, 519-529.

MunaKaTA, Y. (1998). Infant perseveration and implications for object permanence theories: A PDP model of the A-not-B task. Infant Behavior \& Development, 20, 405-416.

Munakata, Y., McClelland, J. L., Johnson, M. H., \& Siegler, R. S. (1997). Rethinking infant knowledge: Toward an adaptive process account of successes and failures in object permanence tasks. Psychological Review, 104, 686-719.

Piaget, J. (1954). The construction of reality in the child. New York: Basic Books.

Smith, L. B., Thelen, E., Titzer, R., \& McLin, D. (1999). Knowing in the context of acting: The task dynamics of the A-not-B error. Psychological Review, 106, 235-260.

Spencer, J. P., Smith, L. B., \& Thelen, E. (2001). Tests of a dynamic systems account of the A-not-B error: The influence of prior experience on the spatial memory abilities of 2-year-olds. Child Development, 72, 1327-1346.

Thelen, E., Schöner, G., Scheier, C., \& Smith, L. B. (2001). The dynamics of embodiment: A field theory of infant perseverative reaching. Behavioral \& Brain Sciences, 24, 1-86.

Wellman, H. M., Cross, D., \& Bartsch, K. (1987). Infant search and object permanence: A meta-analysis of the A-not-B error. Monographs of the Society for Research in Child Development, 51(Whole No. 214).

(Manuscript received June 7, 2006;

revision accepted for publication September 22, 2006.) 